\title{
STRUCTURAL-PARAMETRIC MODEL OF ELECTROELASTIC ACTUATOR FOR MECHATRONICS DEVICES OF NANOTECHNOLOGY
}

\section{SERGEY M. AFONIN}

Department of Intellectual Technical Systems

National Research University of Electronic Technology

MIET

Moscow

Russia

e-mail: eduems@mail.ru

\begin{abstract}
The generalized structural-parametric model, the generalized matrix transfer function of the electroelastic actuator with the output parameters displacements are determined. The parametric structural schematic diagram and the transfer functions of the electroelastic actuator are obtained for calculation of the mechatronics devices of nanotechnology. The structural-parametric models of the piezoactuator for the transverse, longitudinal, shift piezoelectric effects are constructed. The dynamic and static characteristics of the piezoactuator with the output parameter displacement are obtained.
\end{abstract}

Keywords and phrases: electroelastic actuator, piezoactuator, deformation, structuralparametric model, parametric structural schematic diagram, transfer functions.

Received November 20, 2017

(C) 2018 Scientific Advances Publishers 


\section{Introduction}

The electroelastic actuator on the piezoeffect and the electrostriction effect is used for precise alignment in the nanotechnology and the adaptive optics [1-32]. The piezoactuator uses the inverse piezoeffect and serves for the actuation of mechanisms or the management and converts the electrical signals into the displacement and the force [1-8]. The piezoactuator is applied for the drives of the scanning tunneling microscopes, scanning force microscopes and atomic force microscopes [14-32].

Let us consider the generalized structural-parametric model and the generalized parametric structural schematic diagram of the electroelastic actuator are constructed by solving the wave equation with the Laplace transform for the equation of the electromagnetolasticity, the boundary conditions on loaded working surfaces of the actuator, the strains along the coordinate axes. The transfer functions and the parametric structural schematic diagrams of the piezoactuator are obtained from the generalized structural-parametric model. In [6,7] was determined the solution of the wave equation of the piezoactuator. In the [14-16, 30, 31] were obtained the structural-parametric models, the schematic diagrams for simple piezoactuator and were transformed to the structuralparametric model of the electroelastic actuator with the output displacement. The structural parametric model of the electroelastic actuator was determined in contrast electrical equivalent circuit types Cady and Mason for calculation of piezoelectric transmitter and receiver [9-11]. In the paper [12] presents the classic analytical two-port lumpedelement model (LEM) types Cady and Mason of the piezoelectric composite circular plate with the output pressure. In the paper [13] considers the development of various lumped-element models as practical tools to design and manufacture the actuators with the output velocity. In $[8,27]$ was used the transfer functions of the piezoactuator for the decision problem absolute stability conditions for a system controlling the deformation of the electroelastic actuator. The elastic compliances and the mechanical and adjusting characteristics of the piezoactuator were found in [18, 21-23, 28, 29] for calculation its transfer functions and the 
structural-parametric models. The structural-parametric model of the multilayer and compound piezoactuator was determined in [18-20] with the output displacement. In this paper is solving the problem of building the generalized structural parametric model and the generalized parametric structural schematic diagram of the electroelastic actuator for the equation of the electroelasticity. The difference of this work from the papers [30-36] is that the construction of the structure-parametric model of the electroelastic actuator is produced immediately in the general form, and not by the method of mathematical induction from the individual examples for the models of the piezoactuators.

\section{Structural-Parametric Model and Transfer Functions of Electroelastic Actuator}

Let us consider the general structural-parametric model and the parametric structural schematic diagram of the electroelastic actuator with the output parameter displacement. For the electroelastic actuator are presented six stress components $T_{1}, T_{2}, T_{3}, T_{4}, T_{5}, T_{6}$, where the components $T_{1}-T_{3}$ are related to extension compression stresses, $T_{4}-T_{6}$ to shear stresses. In the electroelastic actuator its deformation corresponds to stressed state. For polarized piezoceramics PZT, the matrix state equations $[12,14]$ with the electric and elastic variables have the form two equations, where the first equation represents the direct piezoeffect, the second describes the inverse piezoeffect

$$
\begin{gathered}
\mathbf{D}=\mathbf{d} \mathbf{T}+\varepsilon^{T} \mathbf{E}, \\
\mathbf{S}=\mathbf{s}^{E} \mathbf{T}+\mathbf{d}^{t} \mathbf{E},
\end{gathered}
$$

where $\mathbf{D}$ is the column matrix of electric induction; $\mathbf{S}$ is the column matrix of relative deformations; $\mathbf{T}$ is the column matrix of mechanical

stresses; $\mathbf{E}$ is the column matrix of electric field strength; $\mathbf{s}^{E}$ is the elastic compliance matrix for $E=$ const; $\varepsilon^{T}$ is the matrix of dielectric constants for $T=$ const; $\mathbf{d}^{t}$ is the transposed matrix of the piezoelectric modules. 
The piezoactuator (piezoplate) has the following properties: $\delta$ is the thickness, $h$ is the height, $b$ is the width, respectively $l=\{\delta, h, b$ the working length of the piezoactuator for the longitudinal, transverse and shift piezoeffect. The direction of the polarization axis $P$, i.e., the direction along which polarization was performed, is usually taken as the direction of axis 3 . The equation of the inverse piezoeffect for controlling voltage $[6,12]$ has the form

$$
\begin{gathered}
S_{i}=d_{m i} \Psi_{m}(t)+s_{i j}^{\Psi} T_{j}(x, t), \\
S_{i}=\partial \xi(x, t) / \partial x, \quad \Psi_{m}(t)=E_{m}(t)=U(t) / \delta
\end{gathered}
$$

where $S_{i}$ is the relative displacement of the cross section of the piezoactuator along axis $i, \Psi_{m}(t)$ is the control parameter along axis $m, \xi(x, t)$ is the displacement of the section of the piezoactuator, $d_{m i}$ is the piezomodule, $E_{m}(t)$ is the electric field strength along axis $m, U(t)$ is the voltage between the electrodes of actuator, $s_{i j}^{\Psi}$ is the elastic compliance, $T_{j}$ is the mechanical stress along axis $j$ and $i, j=1,2, \ldots, 6$; $m=1,2,3$. The main size $l=\{\delta, h, b$ for the piezoactuator, respectively, the thickness, the height, the width for the longitudinal, transverse, shift piezoeffect.

For calculation of the electroelastic actuator is used the wave equation $[6,7,12,14]$ for the wave propagation in a long line with damping but without distortions. After Laplace transform is obtained the linear ordinary second-order differential equation with the parameter $p$, where the original problem for the partial differential equation of hyperbolic type using the Laplace transform is reduced to the simpler problem $[6,13]$ for the linear ordinary differential equation

$$
\frac{d^{2} \Xi(x, p)}{d x^{2}}-\gamma^{2} \Xi(x, p)=0
$$


with its solution

$$
\Xi(x, p)=C e^{-x \gamma}+B e^{x \gamma},
$$

where $\Xi(x, p)$ is the Laplace transform of the displacement of the section of the electroelastic actuator, $\gamma=p / c^{\Psi}+\alpha$ is the propagation coefficient, $c^{\Psi}$ is the sound speed for $\Psi=$ const, $\alpha$ is the damping coefficient, $\Psi$ is the control parameter: $E$ for the voltage control, $D$ for the current control, $H$ for the magnet field strength control. From (3), (5), the boundary conditions on loaded surfaces, the strains along the axes the system of equations for the generalized structural-parametric model and the generalized parametric structural schematic diagram are determined for Figure 1 of the actuator with the output parameters the Laplace transform for the displacements $\Xi_{1}(p), \Xi_{2}(p)$ for the faces of the electroelastic actuator in the form

$$
\begin{gathered}
\Xi_{1}(p)=\left(\frac{1}{M_{1} p^{2}}\right)\left\{-F_{1}(p)+\left(\frac{1}{\chi_{i j}^{\Psi}}\right)\left[\begin{array}{l}
d_{m i} \Psi_{m}(p)- \\
\left(\frac{\gamma}{\operatorname{sh}(l \gamma)}\right)\left[\operatorname{ch}(l \gamma) \Xi_{1}(p)-\Xi_{2}(p)\right]
\end{array}\right]\right\}, \\
\Xi_{2}(p)=\left(\frac{1}{M_{2} p^{2}}\right)\left\{-F_{2}(p)+\left(\frac{1}{\chi_{i j}^{\Psi}}\right)\left[\begin{array}{l}
d_{m i} \Psi_{m}(p)- \\
\left.\left.\left(\frac{\gamma}{\operatorname{sh}(l \gamma)}\right)\left[\operatorname{ch}(l \gamma) \Xi_{2}(p)-\Xi_{1}(p)\right]\right]\right\}, \quad(5)
\end{array}\right.\right. \\
\text { where } \quad \chi_{i j}^{\Psi}=\frac{s_{i j}^{\Psi}}{S_{0}}, \quad d_{m i}=\left\{\begin{array}{l}
d_{33}, d_{31}, d_{15} \\
g_{33}, g_{31}, g_{15},
\end{array} \Psi_{m}=\left\{\begin{array}{l}
E_{3}, E_{3}, E_{1} \\
D_{3}, D_{3}, D_{1}
\end{array}\right.\right. \\
s_{i j}^{\Psi}=\left\{\begin{array}{l}
s_{33}^{E}, s_{11}^{E}, s_{55}^{E} \\
s_{33}^{D}, s_{11}^{D},
\end{array} s_{55}^{D}, \quad l=\left\{\delta, h, b, \quad c^{\Psi}=\left\{c^{E}, c^{D}, \gamma^{\Psi}=\left\{\gamma^{E}, \gamma^{D}, d_{m i}\right.\right.\right.\right.
\end{gathered}
$$

is the coefficient of the electroelasticity (piezomodule), $F_{1}(p), F_{2}(p)$ are the Laplace transform of the forces on the faces. Figure 1 shows the generalized parametric structural schematic diagram of the electroelastic actuator corresponding to the set of Equations (6) for the Laplace transform of the displacements of the faces. The generalized transfer 
functions of the of the electroelastic actuator are the ratio of the Laplace transform of the displacement of the face actuator and the Laplace transform of the corresponding control parameter or the force at zero initial conditions. From (6), the generalized matrix equation of the transfer functions of electroelastic actuator has the form

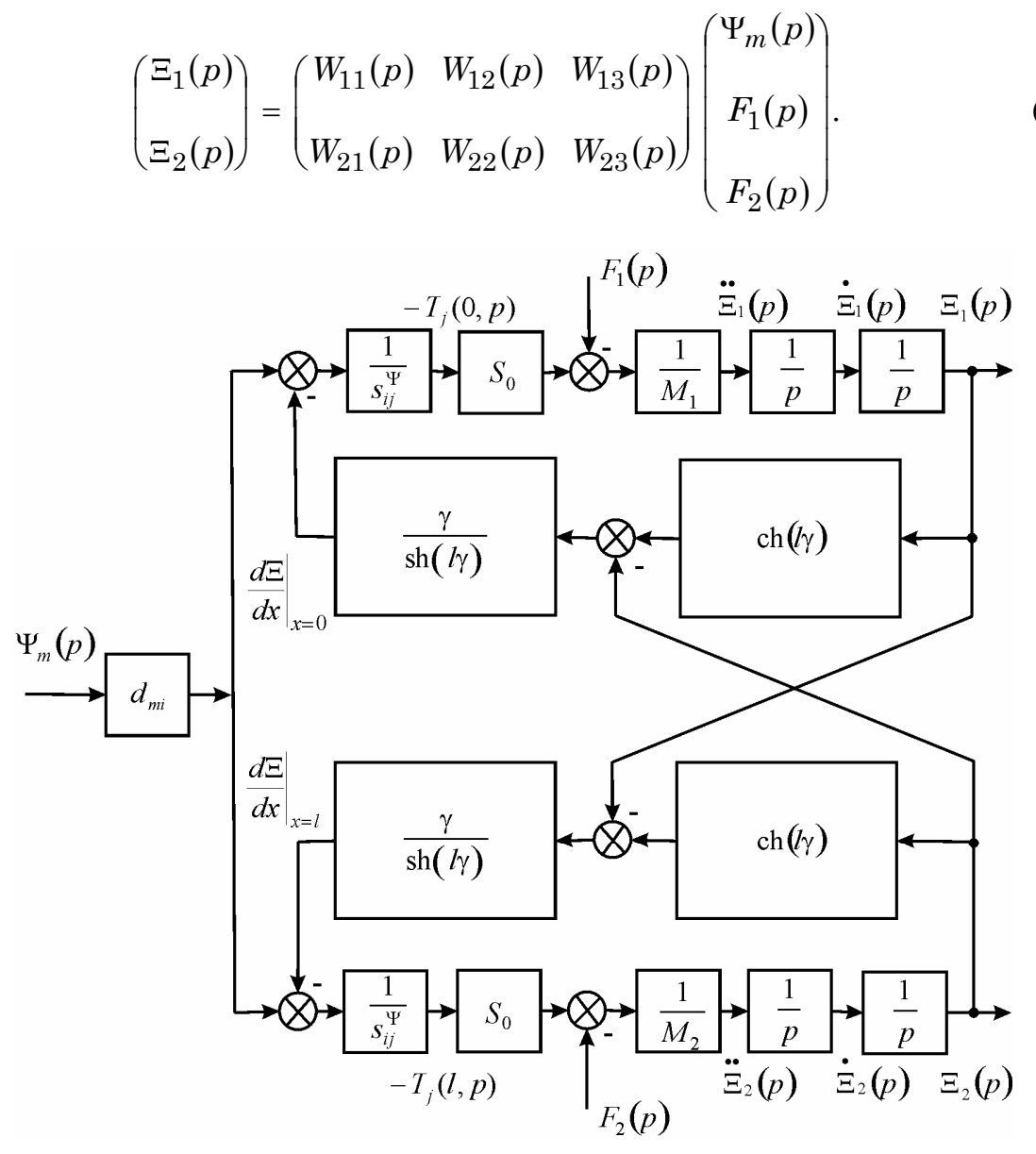

Figure 1. Generalized parametric structural schematic diagram of the of the electroelastic actuator. 
Let us consider the static displacements of the faces for the electroelastic actuator with the output parameter displacement. For $m \ll M_{1}$ and $m \ll M_{2}$, the static displacements of the faces of the piezoactuator for the transverse piezoeffect are obtained from (6) in the form

$$
\begin{aligned}
& \xi_{1}(\infty)=\lim _{\substack{p \rightarrow 0 \\
\alpha \rightarrow 0}} \frac{p W_{11}(p) U_{0}}{\delta p}=\frac{d_{31} h U_{0} M_{2}}{\delta\left(M_{1}+M_{2}\right)}, \\
& \xi_{2}(\infty)=\lim _{\substack{p \rightarrow 0 \\
\alpha \rightarrow 0}} \frac{p W_{21}(p) U_{0}}{\delta p}=\frac{d_{31} h U_{0} M_{1}}{\delta\left(M_{1}+M_{2}\right)} .
\end{aligned}
$$

For the piezoactuator from PZT under the transverse piezoeffect at $m<<M_{1}$ and $m<<M_{2}, d_{31}=2.5 \cdot 10^{-10} \mathrm{~m} / \mathrm{V}, h / \delta=20, U=300 \mathrm{~V}$, $M_{1}=2 \mathrm{~kg}$ and $M_{2}=8 \mathrm{~kg}$ the static displacements of the faces are determined $\xi_{1}(\infty)=1200 \mathrm{~nm}, \xi_{2}(\infty)=300 \mathrm{~nm}, \xi_{1}(\infty)+\xi_{2}(\infty)=1500 \mathrm{~nm}$.

For the approximation of the hyperbolic cotangent by two terms of the power series in transfer function (6), the following expressions of the transfer function of the piezoactuator with one fixed face is obtained for the elastic-inertial load at $M_{1} \rightarrow \infty, m \ll M_{2}$ under the transverse piezoeffect and control voltage for the resistance $R=0$ of the voltage source (Figure 2) in the form

$$
\begin{gathered}
W(p)=\frac{\Xi_{2}(p)}{U(p)}=\frac{d_{31} h / \delta}{\left(1+C_{e} / C_{11}^{E}\right)\left(T_{t}^{2} p^{2}+2 T_{t} \xi_{t} p+1\right)} \\
T_{t}=\sqrt{M_{2} /\left(C_{e}+C_{11}^{E}\right)}, \quad \xi_{t}=\alpha h^{2} C_{11}^{E} /\left(3 c^{E} \sqrt{M\left(C_{e}+C_{11}^{E}\right)}\right)
\end{gathered}
$$

where $U(p)$ is the Laplace transform of the voltage, $T_{t}$ is the time constant, and $\xi_{t}$ is the damping coefficient of the piezoactuator. 


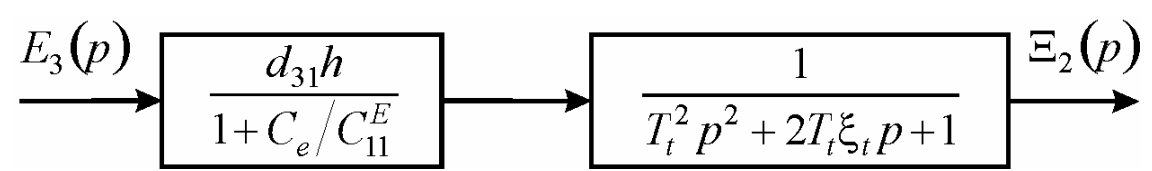

Figure 2. Parametric structural schematic diagram of the voltagecontrolled piezoactuator with one fixed face under transverse piezoeffect for elastic-inertial load.

Let us consider the transient response of the electroelastic actuator with the output parameter displacement. The expression for the transient response of the voltage-controlled piezoactuator for the elasticinertial load under the transverse piezoeffect is determined

$$
\begin{gathered}
\xi(t)=\xi_{m}\left[\begin{array}{c}
-\frac{\xi_{t} t}{T_{t}} \\
1-\frac{e}{\sqrt{1-\xi_{t}^{2}}} \sin \left(\omega_{t} t+\varphi_{t}\right)
\end{array}\right], \\
\xi_{m}=\frac{d_{31}(h / \delta) U_{m}}{1+C_{e} / C_{11}^{E}}, \omega_{t}=\frac{\sqrt{1-\xi_{t}^{2}}}{T_{t}}, \varphi_{t}=\operatorname{arctg}\left(\frac{\sqrt{1-\xi_{t}^{2}}}{\xi_{t}}\right),
\end{gathered}
$$

where $\xi_{m}$ is the steady-state value of displacement of the piezoactuator, $U_{m}$ is the amplitude of the voltage. For the voltage-controlled piezoactuator from the piezoceramics PZT under the transverse piezoelectric effect for the elastic-inertial load $M_{1} \rightarrow \infty, m \ll M_{2}$ and input voltage with amplitude $U_{m}=250 \mathrm{~V}$ at $d_{31}=2.5 \cdot 10^{-10} \mathrm{~m} / \mathrm{V}$, $h / \delta=20, M_{2}=9 \mathrm{~kg}, C_{11}^{E}=2 \cdot 10^{7} \mathrm{~N} / \mathrm{m}, C_{e}=0.5 \cdot 10^{7} \mathrm{H} / \mathrm{m}$ are obtained values $\xi_{m}=1000 \mathrm{~nm}, T_{t}=0.6 \cdot 10^{-3} \mathrm{c}$.

\section{Results and Discussions}

The electroelastic actuator solves problem of precise matching, compensation of temperature and gravitational deformations in the mechatronics devices of the nanotechnology. The structural-parametric 
model and parametric structural schematic diagrams of the voltagecontrolled piezoactuator for the longitudinal, transverse, and shift piezoelectric effects are determined from the generalized structuralparametric model of the electroelastic actuator with the replacement of the generalized parameters on the parameters of the piezoactuator.

The transfer functions in matrix form are describe deformations of the electroelastic actuator during its operation as a part of the mechatronics devices of the nanotechnology.

The generalized structural-parametric model, the generalized parametric structural schematic diagram and the matrix equation of the electroelastic actuator with the output parameters displacements are obtained from the solutions of the wave equation with the Laplace transform and from its deformations along the coordinate axes. From the generalized matrix equation for the transfer functions of the electroelastic actuator after algebraic transformations are constructed the matrix equations of the piezoactuator for the longitudinal, transverse, and shift piezoelectric effects.

\section{Conclusion}

The generalized structural-parametric model, the generalized parametric structural schematic diagram, and the matrix equation of the electroelastic actuator with the output parameters displacements for the mechatronics devices of nanotechnology are obtained.

The structural-parametric model, the matrix equation, and the parametric structural schematic diagram of the piezoactuator for the transverse, longitudinal, and shift piezoelectric effects are determined from the generalized structural-parametric model of the electroelastic actuator for nanotechnology. 
From the solution of the wave equation, from the equation of the electroelasticity and the deformations along the coordinate axes the generalized structural-parametric model and the generalized parametric structural schematic diagram of the electroelastic actuator with the output parameters displacements are constructed for the nanotechnology. The deformations of the electroelastic actuator are described by the matrix equation for the transfer functions of the actuator.

\section{References}

[1] J. Schultz, J. Ueda and H. Asada, Cellular Actuators, Butterworth-Heinemann Publisher: Oxford (2017), 382.

[2] K. Uchino, Piezoelectric Actuator and Ultrasonic Motors, Kluwer Academic Publisher: Boston MA (1997), 347.

[3] J. Przybylski, Static and dynamic analysis of a flextensional transducer with an axial piezoelectric actuation, Engineering Structures 84 (2015), 140-151.

DOI: https://doi.org/10.1016/j.engstruct.2014.11.025

[4] J. Ueda, T. W. Secord and H. H. Asada, Large effective-strain piezoelectric actuators using nested cellular architecture with exponential strain amplification mechanisms, IEEE/ASME Transactions on Mechatronics 15(5) (2010), 770-782.

DOI: https://doi.org/10.1109/TMECH.2009.2034973

[5] M. Karpelson, G.-Y. Wei and R. J. Wood, Driving high voltage piezoelectric actuators in microrobotic applications, Sensors and Actuators A: Physical 176 (2012), 78-89.

DOI: https://doi.org/10.1016/j.sna.2011.11.035

[6] S. M. Afonin, Solution of the wave equation for the control of an electromagnetoelastic transducer, Doklady Mathematics 73(2) (2006), 307-313.

DOI: https://doi.org/10.1134/S1064562406020402

[7] S. M. Afonin, Structural parametric model of a piezoelectric nanodisplacement transduser, Doklady Physics 53(3) (2008), 137-143.

DOI: https://doi.org/10.1134/S1028335808030063

[8] S. M. Afonin, Stability of strain control systems of nano- and microdisplacement piezotransducers, Mechanics of Solids 49(2) (2014), 196-207.

DOI: https://doi.org/10.3103/S0025654414020095 
[9] V. Talakokula, S. Bhalla, R. J. Ball, C. R. Bowen, G. L. Pesce, R. Kurchania, B. Bhattacharjee, A. Gupta and K. Paine, Diagnosis of carbonation induced corrosion initiation and progression in reinforced concrete structures using piezo-impedance transducers, Sensors and Actuators A: Physical 242 (2016), 79-91.

DOI: https://doi.org/10.1016/j.sna.2016.02.033

[10] W. G. Cady, Piezoelectricity An Introduction to the Theory and Applications of Electromechancial Phenomena in Crystals, McGraw-Hill Book Company, New York, London (1946), 806.

[11] Physical Acoustics: Principles and Methods, Vol. 1. Part A. Methods and Devices, Editor W. Mason, New York: Academic Press (1964), 515.

[12] S. Prasad, Q. Gallas, S. Horowitz, B. Homeijer et al., Analytical electroacoustic model of a piezoelectric composite circular plate, AIAA Journal 41(10) (2006), 2311-2318.

DOI: https://doi.org/10.2514/1.19855

[13] M. Chiatto, F. Capuano, G. Coppola and L. de Luca, LEM characterization of synthetic jet actuators driven by piezoelectric element: A review, Sensors 17(6) (2017), 1216-1246.

DOI: https://doi.org/10.3390/s17061216

[14] S. M. Afonin, Structural-parametric model and transfer functions of electroelastic actuator for nano- and microdisplacement, Chapter 9 in Piezoelectrics and Nanomaterials: Fundamentals Developments and Applications, Editor I. A. Parinov, Nova Science: New York (2015), 225-242.

[15] S. M. Afonin, Structural-parametric model electromagnetoelastic actuator nano- and microdisplacement for precision engineering, Engineering and Technology 3(6) (2016), 110-119.

[16] S. M. Afonin, Structural-parametric models and transfer functions of electromagnetoelastic actuators nano- and microdisplacement for mechatronic systems, International Journal of Theoretical and Applied Mathematics 2(2) (2016), 52-59.

DOI: https://doi.org/10.11648/j.ijtam.20160202.15

[17] S. M. Afonin, Parametric structural diagram of a piezoelectric converter, Mechanics of Solids 37(6) (2002), 85-91.

[18] S. M. Afonin, Deformation, fracture, and mechanical characteristics of a compound piezoelectric transducer, Mechanics of Solids 38(6) (2003), 78-82.

[19] S. M. Afonin, Parametric block diagram and transfer functions of a composite piezoelectric transducer, Mechanics of Solids 39(4) (2004), 119-127.

[20] S. M. Afonin, Generalized parametric structural model of a compound elecromagnetoelastic transduser, Doklady Physics 50(2) (2005), 77-82.

DOI: https://doi.org/10.1134/1.1881716 
[21] S. M. Afonin, Design static and dynamic characteristics of a piezoelectric nanomicrotransducers, Mechanics of Solids 45(1) (2010), 123-132.

DOI: https://doi.org/10.3103/S0025654410010152

[22] S. M. Afonin, Electromechanical deformation and transformation of the energy of a nano-scale piezomotor, Russian Engineering Research 31(7) (2011), 638-642.

DOI: https://doi.org/10.3103/S1068798X11070033

[23] S. M. Afonin, Electroelasticity problems for multilayer nano- and micromotors, Russian Engineering Research 31(9) (2011), 842-847.

DOI: https://doi.org/10.3103/S1068798X11090036

[24] S. M. Afonin, Nano- and micro-scale piezomotors, Russian Engineering Research 32(7-8) (2012), 519-522.

DOI: https://doi.org/10.3103/S1068798X12060032

[25] S. M. Afonin, Optimal control of a multilayer submicromanipulator with a longitudinal piezo effect, Russian Engineering Research 35(12) (2015), 907-910.

DOI: https://doi.org/10.3103/S1068798X15120035

[26] S. M. Afonin, Block diagrams of a multilayer piezoelectric motor for nano- and microdisplacements based on the transverse piezoeffect, Journal of Computer and Systems Sciences International 54(3) (2015), 424-439.

DOI: https://doi.org/10.1134/S1064230715020021

[27] S. M. Afonin, Absolute stability conditions for a system controlling the deformation of an elecromagnetoelastic transduser, Doklady Mathematics 74(3) (2006), 943-948.

DOI: https://doi.org/10.1134/S1064562406060391

[28] S. M. Afonin, Elastic compliances and mechanical and adjusting characteristics of composite piezoelectric transducers, Mechanics of Solids 42(1) (2007), 43-49.

DOI: https://doi.org/10.3103/S0025654407010062

[29] S. M. Afonin, Static and dynamic characteristics of a multi-layer electroelastic solid, Mechanics of Solids 44(6) (2009), 935-950.

DOI: https://doi.org/10.3103/S0025654409060119

[30] S. M. Afonin, Structural-parametric model electromagnetoelastic actuator nanodisplacement for mechatronics, International Journal of Physics 5(1) (2017), 9-15.

DOI: https://doi.org/10.12691/ijp-5-1-27

[31] S. M. Afonin, A Structural-parametric model of electro elastic actuator for nano- and microdisplacement of mechatronic system, Chapter 8 in Advances in Nanotechnology, Volume 19, Editors Z. Bartul and J. Trenor, Nova Science: New York (2017), 259-284.

[32] Springer Handbook of Nanotechnology, Ed. by B. Bhushan, Springer: Berlin, New York (2004), 1222. 
[33] S. M. Afonin, Structural-parametric model of piezoactuator nano- and microdisplacement for nanoscience, AASCIT Journal of Nanoscience 3(3) (2017), 12-18.

[34] S. M. Afonin, Solution wave equation and parametric structural schematic diagrams of electromagnetoelastic actuators nano- and microdisplacement, International Journal of Mathematical Analysis and Applications 3(4) (2016), 31-38.

[35] S. M. Afonin, Structural-parametric model electromagnetoelastic actuator nano- and microdisplacement for precision engineering, Engineering and Technology 3(6) (2016), 110-119.

[36] S. M. Afonin, Decision wave equation and block diagram of electromagnetoelastic actuator nano- and microdisplacement for communications systems, International Journal of Information and Communication Sciences 1(2) (2016), 22-29.

DOI: https://doi.org/10.11648/j.ijics.20160102.12 